\title{
AS602801, an Anti-Cancer Stem Cell Drug Candidate, Suppresses Gap-junction Communication Between Lung Cancer Stem Cells and Astrocytes
}

\author{
KENTA KURAMOTO $^{1}$, MASAHIRO YAMAMOTO ${ }^{1}$, SHUHEI SUZUKI ${ }^{1,2}$, TOMOMI SANOMACHI ${ }^{1,2}$, \\ KEITA TOGASHI $^{1,3}$, SHIZUKA SEINO ${ }^{1}$, CHIFUMI KITANAKA ${ }^{1,4}$ and MASASHI OKADA ${ }^{1}$ \\ Departments of ${ }^{1}$ Molecular Cancer Science, ${ }^{2}$ Clinical Oncology, and ${ }^{3}$ Ophthalmology, \\ Yamagata University School of Medicine, Yamagata, Japan; \\ ${ }^{4}$ Research Institute for Promotion of Medical Sciences, Yamagata University Faculty of Medicine, Yamagata, Japan
}

\begin{abstract}
Background/Aim: Cancer stem cells (CSCs) are associated with tumorigenesis, recurrence, and metastasis. Cell-cell communication via gap junctions (GJs) between metastatic cancer cells and astrocytes is necessary for brain metastasis. Agents targeting communication between CSCs and astrocytes are expected to suppress brain metastasis. Materials and Methods: Using the A549 CSC, a cancer stem-like cell derived from A549, we examined the effect of AS602801, an anti-cancer stem cell agent whose safety has been confirmed in a phase 2 clinical trial, on GJ communication and connexin expression using a dye-transfer assay and immunoblot analysis, respectively. Results: AS602801 specifically suppressed cell-cell communication in A549 CSCs without any suppression of GJ communication in astrocytes; it also decreased the expression of connexin 43, a constituent of GJs, in A549 CSCs. Conclusion: The anti-cancer stem cell agent, AS602801, is a potential drug candidate against brain metastasis.
\end{abstract}

Metastatic brain tumors are one of the most intractable tumors and are frequently found at metastatic sites. Several types of cancers including lung cancer, breast cancer, and melanoma can spread to the brain (1-3). Among them, the lung is the most common (40-50\%) primary site with 25 $40 \%$ of non-small cell lung carcinoma (NSCLC) patients showing brain metastasis (4). Cancer metastasis proceeds

Correspondence to: Masashi Okada and Chifumi Kitanaka, Department of Molecular Cancer Science, Yamagata University School of Medicine, Yamagata, 990-9585, Japan. Tel: +81 236285214, Fax: +81 236285215, e-mail: m-okada@med.id.yamagata-u.ac.jp (M.O.), ckitanak@med.id.yamagata-u.ac.jp (C.K.)

Key Words: Cell-cell interaction, CSC, astrocytes, metastasis, AS602801. through a multistep process. When cancer cells metastasize to the brain, cross-talk between the metastatic cancer cells and astrocytes plays a pivotal role. The metastatic cancer cells communicate with astrocytes through gap junctions (GJs) that directly link the cytoplasm of the two cells $(5,6)$. Cell-cell communication via GJs has been reported to regulate brain metastasis at various steps (6-9). It has recently been reported that GJs consisting of connexin 43 (Cx43), the principal GJ constituent protein in epithelial lung cancer cells and astrocytes, promote brain metastasis of cancer cells via cGAMP transfer between cancer cells and astrocytes (5). Cancer stem cells (CSCs) contribute significantly to the establishment of metastasis $(10,11)$. A GJ inhibitor, oleamide, has been found to suppress metastatic foci of MDA-MB-231 cells intravenously injected into mice, resulting in increased survival (12). The oral drugs tonabersat, a neurogenic inflammation inhibitor and meclofenamate, a non-steroidal anti-inflammatory drug (NSAID), inhibit dye transfer activity, paracrine signal transduction, and brain metastasis in a breast cancerxenograft model (5). However, the effect of drugs targeting CSCs by inhibiting their GJ-communication and metastasis is not reported. Such drugs are strongly expected to suppress brain metastasis.

AS602801 (also known as bentamapimod) is a novel JNK inhibitor originally developed during a search for drugs to treat neurological disorders (13). The efficacy of AS602801 against endometriosis has been confirmed in animal models $(14,15)$. A phase 2 clinical trial (NCT 01630252) in patients with inflammatory endometriosis has been completed to confirm its efficacy and safety. We demonstrated that AS602801 inhibits CSCs in vitro and in vivo, and AS602801 is a candidate anti-cancer drug (16). In the present study, we examined the effects of AS602801 on GJs in CSCs to determine whether it can be a promising drug against brain metastasis. 


\section{Materials and Methods}

Reagents and antibodies. AS602801 was purchased from AdooQ BioScience (Irvine, CA, USA) and dissolved in dimethyl sulfoxide (DMSO) to prepare a $10 \mathrm{mM}$ stock solution. Anti-Connexin 43 (\#3512), anti-c-Jun (\#9165), anti-phospho-c-Jun (Ser 63) (\#9261) and anti-GAPDH (\#5174) antibodies were purchased from Cell Signaling Technology, Inc. (Danvers, MA, USA). Horseradish peroxidase (HRP)-conjugated secondary antibodies were purchased from Jackson ImmunoResearch (West Grove, PA, USA).

Cell culture. A549 CSCs used in this study have been previously described $(16,17)$. A549 CSCs were maintained under monolayer stem cell culture condition $(16,17)$. Briefly, cells were cultured on collagen type I-coated dishes (IWAKI, Tokyo, Japan) in stem cell culture medium (DMEM/F-12 supplemented with 1\% B27 [Thermo Fisher Scientific, Waltham, MA, USA], $20 \mathrm{ng} / \mathrm{ml}$ epidermal growth factor (EGF) and fibroblast growth factor (FGF) 2 [Peprotech, Inc., Rocky Hill, NJ, USA], D-(+)-glucose [final concentration $26.2 \mathrm{mM}$ ], L-glutamine [final concentration $4.5 \mathrm{mM}$ ], 100 units $/ \mathrm{ml}$ penicillin and $100 \mu \mathrm{g} / \mathrm{ml}$ streptomycin). The stem cell culture medium was changed every three days, and EGF and FGF2 were added to the medium every day. Mouse primary astrocytes were isolated from the cerebral cortex of neonatal mice. Collected cerebral cortical tissues were rinsed with ice-cold phosphate buffered saline (PBS) and mechanically dissociated by gentle pipetting. Dissociated cells, including astrocytes, were plated on collagen I-coated dishes after passing through a $70-\mu \mathrm{m}$ cell strainer. Thirty min after seeding, the dishes were shaken, and the medium was changed to improve the purity of astrocytes. Medium of cells was replaced with fresh medium every $72 \mathrm{~h}$ and used for experiments 7-14 days after seeding. Cultures with more than $70 \%$ of cells positive for GFAP were considered pure astrocyte cultures (not shown). Mouse primary astrocytes and parental A549 cells were maintained in DMEM medium supplemented with $10 \%$ FBS, 100 units $/ \mathrm{ml}$ penicillin, and $100 \mu \mathrm{g} / \mathrm{ml}$ streptomycin.

RT-PCR. Total RNA was isolated from treated A549 CSCs using TRIzol reagent (Thermo Fisher Scientific), following the manufacturer's protocol. For first strand cDNA synthesis, total RNA was reverse transcribed using random hexamer primers and PrimeScript II RTase (Takara Bio, Inc., Tokyo, Japan). Target genes were amplified with Quick Taq HS DyeMix (TOYOBO CO., LTD., Tokyo, Japan) using the following gene-specific primer sets; 5'-TCTTTCATTAGGGGG AAGGCG-3' (Forward primer) and 5'-GGAGTTTGCCTAAGG CGCTC-3' (Reverse primer) for Connexin 43; 5'-ACCATCTTCCA GGAGCGAGAT-3' (Forward primer) and 5'-ATGACGAACATGGG GGCATC-3' (Reverse primer) for GAPDH. These gene-specific RTPCR primers were designed using Primer-BLAST (https://www.ncbi.nlm.nih.gov/tools/primer-blast/). The PCR products were resolved via electrophoresis in a $1.5 \%$ agarose gel containing ethidium bromide. Specific bands were detected using a ChemiDoc Touch (Bio-Rad, Hercules, CA, USA). The intensity of specific bands was quantified using the software ImageJ (https://imagej.nih.gov/ij/).

Dye transfer assay. Dye transfer assay was performed based on a previous report (5) with modifications. Donor cells were labeled with $1 \mathrm{mg} / \mathrm{ml}$ calcein-AM (nacalai tesque, Kyoto, Japan), and acceptor cells were labeled with $10 \mu \mathrm{M}$ DiD (Takara) for $30 \mathrm{~min}$. The donor cells were co-cultured with the acceptor cells at a 1:1 ratio for six hours in stem cell culture medium without any drugs, and then subjected to analysis of calcein transfer from donor cells to acceptor cells using flow cytometry. At least $1 \times 10^{4}$ acceptor cells were gated using side and forward scatter to identify viable cell populations, and the rate of dye transfer was evaluated. All analyses were run on the FACSCanto II Flow Cytometer (BD Biosciences, San Jose, CA, USA), and the data were analyzed using the software FlowJo, version 7.6.5 (Treestar Inc., Ashland, OR, USA).

Immunoblot analysis. Immunoblot analysis was conducted as described previously $(18,19)$. Cells were washed with ice-cold PBS and lysed in Laemmli buffer (62.5 mM Tris-HCl [pH 6.8], $2 \%$ SDS, $10 \%$ Glycerol). Cell lysates were boiled at $95^{\circ} \mathrm{C}$ for $5 \mathrm{~min}$, and protein concentration was determined using a BCA protein assay kit (Pierce Biotechnology, Inc., Rockford, IL, USA). After measurement of protein concentration, 2-mercaptoethanol and bromophenol blue was added to each sample at $5 \%$ and $0.002 \%$ concentrations, respectively, and samples were boiled again at $95^{\circ} \mathrm{C}$ for $5 \mathrm{~min}$. Samples containing equivalent amounts were resolved via SDS-PAGE, transferred to polyvinylidene difluoride membranes, and probed with primary antibodies and subsequent HRPconjugated secondary antibodies. Specific bands were visualized using Immobilon Western Chemiluminescent HRP Substrate (Merck Millipore) and detected using a ChemiDoc Touch (Bio-Rad). The intensity of specific bands was quantified using ImageJ.

Statistical analysis. All data are expressed as mean \pm standard deviation (SD). Differences were compared using 2-tailed Student's $t$-test. $p<0.05$ was considered statistically significant and is indicated by an asterisk.

\section{Results}

AS602801 inhibits the function of gap-junctions in cancer stem cells. To examine whether AS602801 affects cell-cell communication in cancer stem cells, its effects were evaluated A549 CSCs, which are derived from the A549 nonsmall lung cancer cell line, using a dye transfer assay. The dye transfer assay involves measurement of calcein fluorescence transferred from calcein-labeled cells (calcein (+) donor cells) to DiD-labeled cells (calcein (-) acceptor cells) using flow cytometry to examine GJ activity (Figure 1A). AS602801 treatment significantly decreased the number of cells with transferred calcein fluorescence, suggesting that it suppressed GJs in the A549 CSCs (Figure 1B). Because GJs in astrocytes are responsible for important homeostatic functions such as $\mathrm{Ca}^{2+}$ waves which are mediated via diffusion of cytoplasmic inositol triphosphate (20), we assessed the effect of AS602801 on GJ communication in primary astrocytes freshly isolated from the mouse cerebral cortex. Unlike A549 CSCs, AS602801 treatment had no effect on the function of GJ-mediated dye transfer rate between the astrocytes (Figure 2). Because communication between cancer cells and the non-tumor astrocytes through GJs plays an important role in brain metastasis $(5,6)$, we next examined whether AS602801 decreases dye transfer rate between A549 CSCs and astrocytes. AS602801 significantly decreased dye transfer rate in both directions, from A549 CSCs to astrocytes 
A

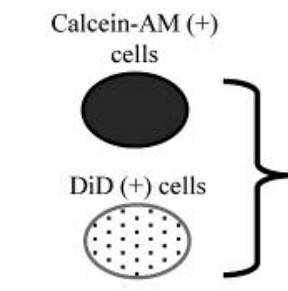

Control or AS treatment for $24 \mathrm{~h}$

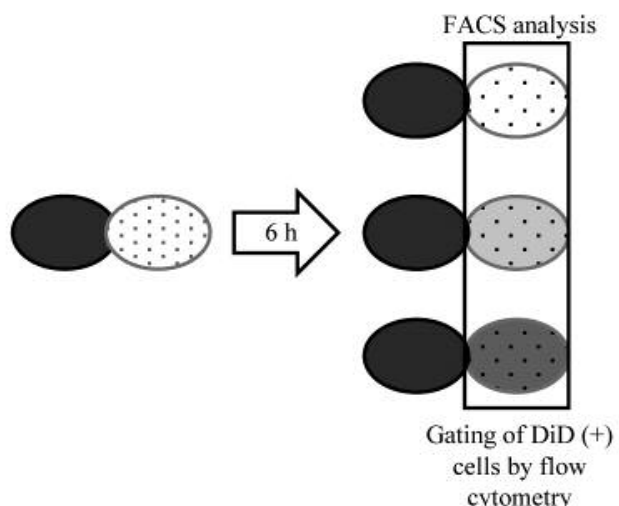

B

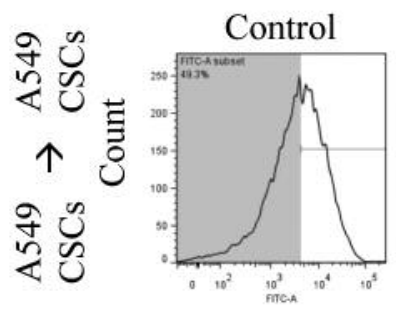

\section{AS602801}

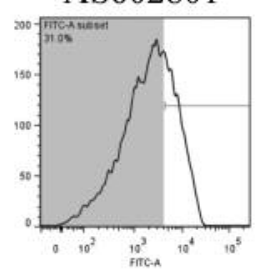

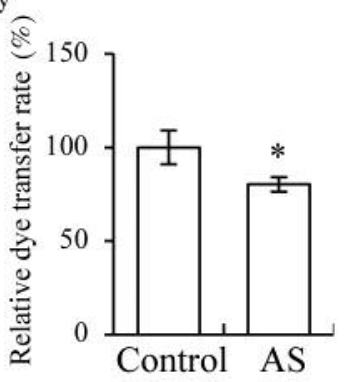

Figure 1. AS602801 suppressed gap junctions in A549 CSC. (A) Schematic model for the study of cell-cell communication using dye transfer. (B) Histograms and quantification of dye transfer between A549 CSCs. A549 CSCs were incubated without or with $7.5 \mu M$ AS602801 AS for 24 h, then subjected to dye transfer assay. Representative histograms are shown. Data are shown as means \pm S.D. from triplicate samples of a representative experiment repeated with similar results. ${ }^{*} p<0.05$.

A
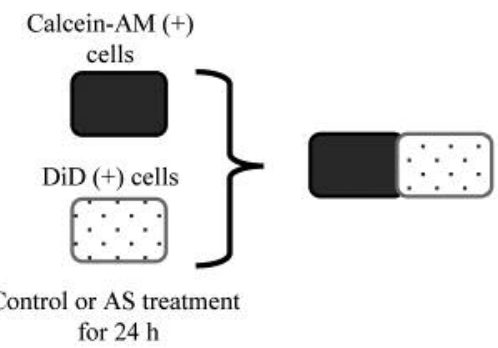

$$
\text { for } 24 \mathrm{~h}
$$

B

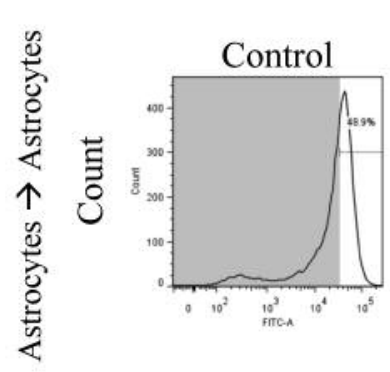

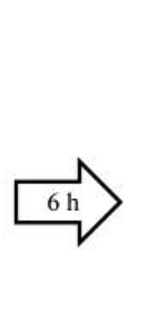

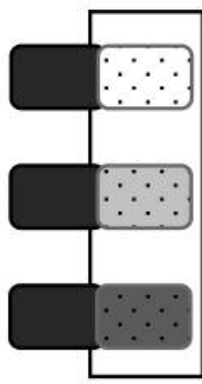

Gating of DiD (+)

cells by flow

cytometry 
(Figure 3A and B) and from astrocytes to A549 CSCs (Figure $3 \mathrm{C}$ and D). Collectively, these results demonstrated that AS602801 could specifically inhibit cell-cell communication via GJs in tumor cells but not in normal astrocytes.

Connexin 43 expression is down-regulated by AS602801. Having shown that AS602801 is a potent inhibitor of GJ function in cancer cells, we next explored the mechanisms by which AS602801 inhibits cell-cell communication in the cells. Recently, it was reported that GJs consisting of connexin 43 (Cx43), the principal GJ constituent protein in epithelial lung cancer cells and astrocytes, promotes brain metastasis of cancer cells via cGAMP transfer between cancer cells and astrocytes (5). Because differences in Cx43-expression status and cell-cell communication between CSCs and non-CSCs of non-small lung cancer are unknown, we examined Cx43 expression levels in A549 CSCs and parental A549 cells. The protein expression of Cx43 in A549 CSCs was approximately two-fold higher than that in parental A549 cells (Figure 4A). In addition, previous reports have shown that $\mathrm{Cx} 43$ expression level is linked to the function of GJs $(5,9)$; therefore, we examined whether GJ communication in CSCs is higher than that in A549 parental cells using a dye transfer assay. Indeed, dye transfer activity in CSCs was significantly higher than that in parental cells (Figure 4B). Because AS602801 impaired GJ communication in cancer cells but not in astrocytes, we expected this agent to suppress the expression of $\mathrm{Cx} 43$. As expected, AS602801 treatment decreased protein expression level of Cx43 in A549 CSCs in a time- and dose-dependent manner (Figure 4C and D). Twenty-four hours after AS602801 treatment, Cx43 mRNA levels were also significantly decreased (Figure 4E). Thus, the results revealed that Cx43 was expressed at higher levels in A549 CSCs than in non-CSCs, and that its expression was suppressed by AS602801.

\section{Discussion}

In NSCLC patients, recurrence and brain metastasis contribute to decreased survival $(21,22)$. Communication between metastatic tumor cells and astrocytes across GJs is important during the late stages of brain metastasis. Once brain-metastatic cells communicate with astrocytes via GJs, the expression patterns of various genes are altered, affecting various signaling pathways which regulate cell growth, stem cell proliferation, migration, cell division, aging, apoptosis, and drug resistance in cancer cells $(6,8)$. GJs are composed of connexins. Cx43 and $\mathrm{Cx} 30$ are the most abundant connexins in the brain $(6,23)$. In particular, $\mathrm{Cx} 43$ is expressed in metastatic tumor tissues at cancer cell-astrocyte interfaces, and its expression level is higher in metastatic tumor tissues than in primary tumor tissues or normal brain tissues (5). CSCs which possess tumorigenic capacity also have a central role in the aggressiveness of tumor metastasis. Indeed, our results in the present study showed that Cx43 expression level in lung CSCs is clearly higher than that in serum-cultured parental (most likely non-stem) cancer cells. Additionally, CSCs showed high dye transfer activity via GJs compared with parental cells. In previous reports, $\mathrm{Cx} 43$ expression level has been found to be linked to high GJcommunication function $(5,9)$. Our results in the present study and previous reports suggest that brain-metastatic potential of CSCs might coincide with high expression of Cx43 in CSCs. Moreover, it was recently reported that transfer of cGAMP from cancer cells to astrocytes through GJs consisting of $\mathrm{Cx} 43$ promoted brain metastasis of cancer cells (5). Therefore, GJs between tumor cells and astrocytes could be a good therapeutic target to treat metastatic cancers.

Notably, we identified AS602801 as a candidate drug for inhibiting GJ-dependent cell-cell communication in CSCs. We found that AS602801 suppresses the expression of $\mathrm{Cx} 43$ in CSCs. Most importantly, this agent does not impair the molecular transport function of astrocyte-astrocyte communications, and we previously reported that AS602801 does not affect IMR90, normal human fibroblasts, at the concentrations used (16). AS602801 is a therapeutic agent in endometriosis treatment, and its molecular mechanism involves ATP-competitive JNK inhibition; its safety was confirmed through a phase 2 clinical trial $(13,24)$. Therefore, AS602801 can be considered a promising therapeutic drug targeting cell-cell communication. However, some reports have shown that $\mathrm{Cx} 43$ in GJs demonstrated a tumor suppressive role in glioblastoma stem cells $(25,26)$. Indeed, because other reports show that $\mathrm{Cx} 43$ expression positively regulates E-cadherin expression, downregulation of $\mathrm{Cx} 43$ might promote the initial migration step in metastasis (2628). Therefore, timing of treatment may be important in the development of a treatment regimen.

Previously, we reported that AS602801 inhibits CSCs in vitro and in vivo without significant side effects (16). Additionally, we found that AS602801 suppresses the expression of $\mathrm{Cx} 43$ in A549 CSCs. It remains unknown whether other anti-CSC agents suppress CSC capacity, Cx43 expression, and/or GJ-dependent communication. However, because it is possible to reduce CSC capacity in (metastatic) tumor cells and suppress cell-cell communication in latestage metastasis, AS602801 could be a therapeutic agent against highly brain-metastatic CSCs. AS602801 is a JNK inhibitor and several previous reports have shown that activation of the JNK pathway induces Cx43 expression in several cells including cancer cells and normal somatic cells (2, 29-31). Although the relationship between the JNK signaling pathway and Cx43 in CSCs is not well known, AS602801 was found to suppress Cx43 expression in A549 CSCs in the present study. STRING analysis (https://stringdb.org/) showed that $\mathrm{Cx} 43$ has a putative interaction with JNK pathway genes such as jun and fos. Future studies might clarify whether the JNK signaling pathway is linked to Cx43/GJ-dependent cell-cell communication. 
A
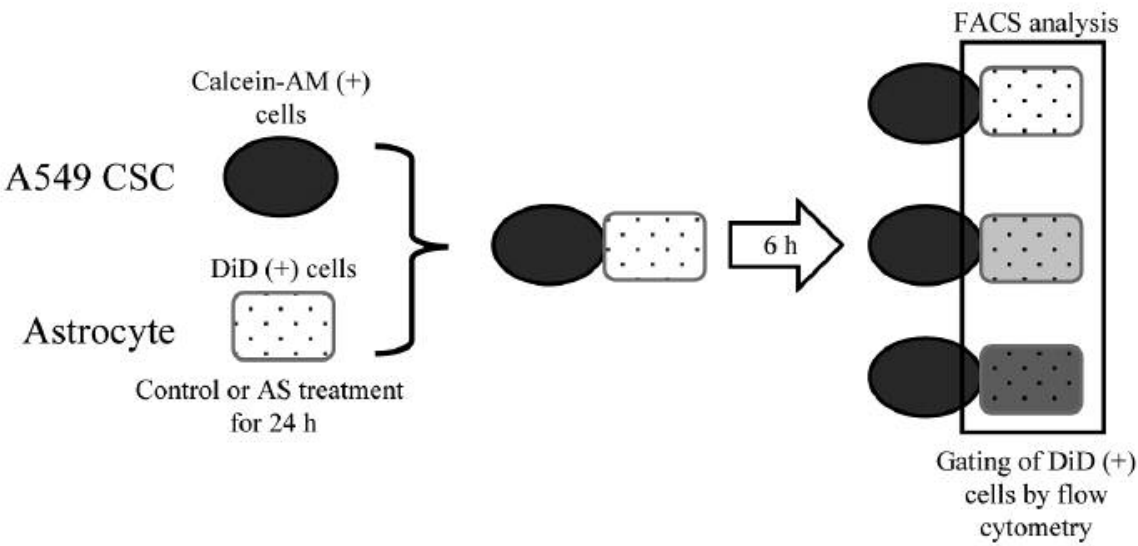

B
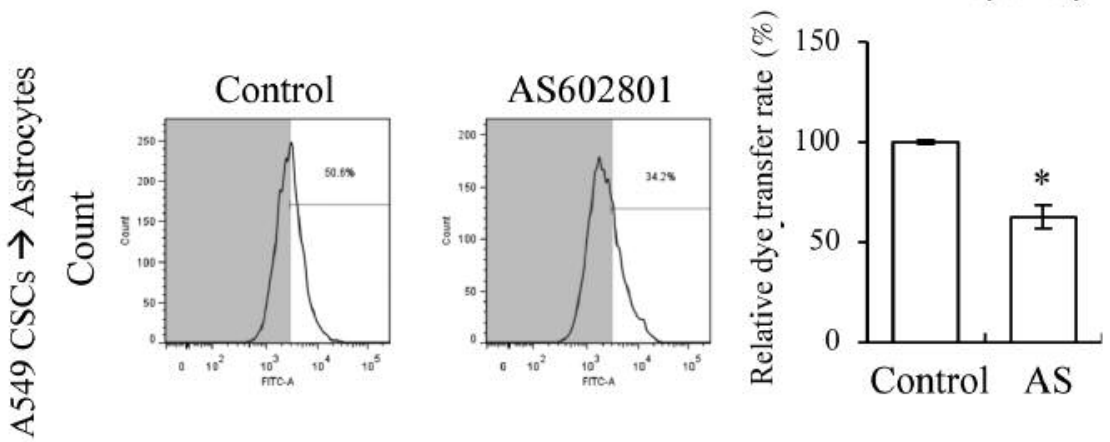

C
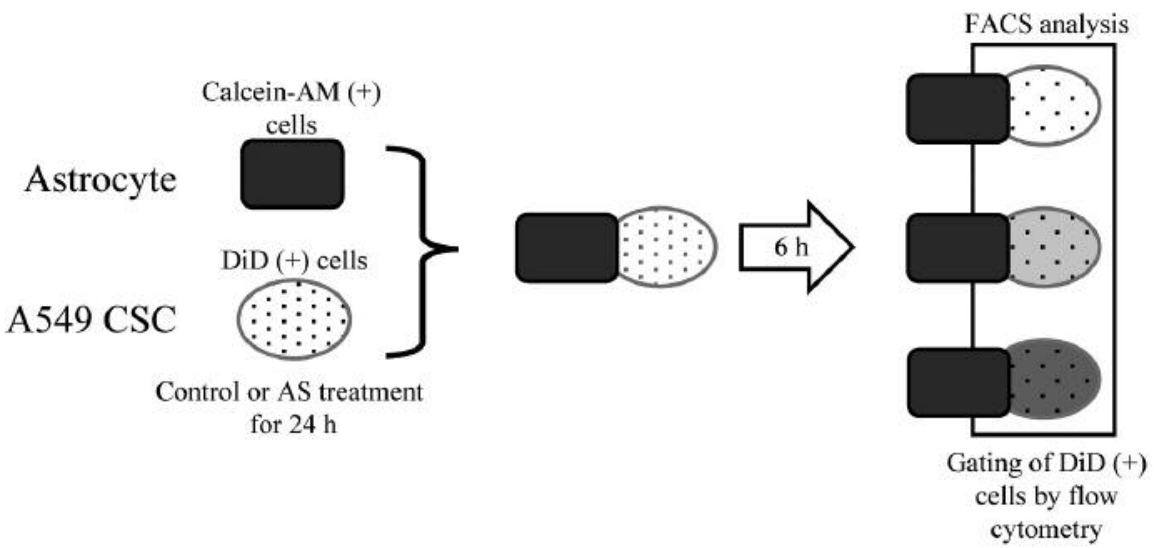

D
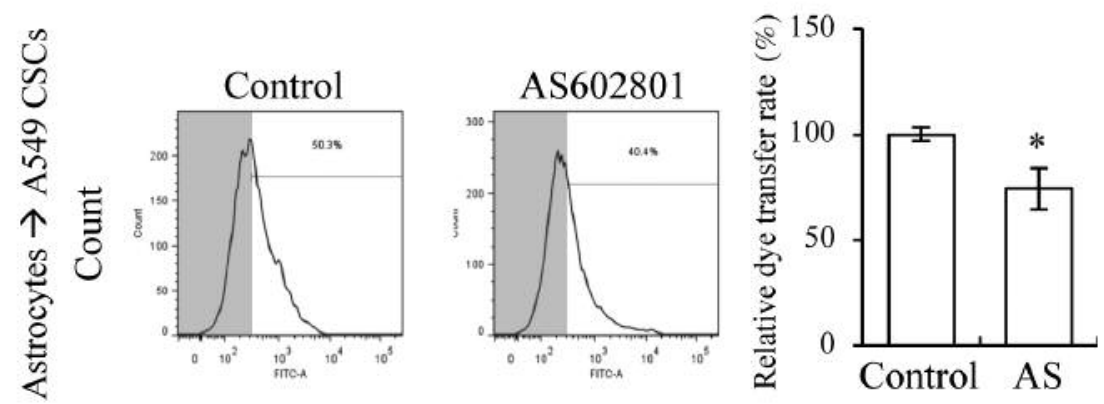

Figure 3. AS602801 inhibited the function of A549 CSC-astrocyte gap junction. (A, C) Schemes of dye transfer analyses of A549 CSC-astrocyte or astrocyte-A549 CSC communication. (B, D) Histograms and quantification of dye transfer between A549 CSCs and astrocytes. A549 CSCs and astrocytes were incubated without or with $7.5 \mu \mathrm{M}$ AS602801 AS for $24 \mathrm{~h}$ and then subjected to dye transfer assay. Representative histograms are shown. Data are shown as means $\pm S . D$. from triplicate samples of a representative experiment repeated with similar results. ${ }^{*} p<0.05$. 
A

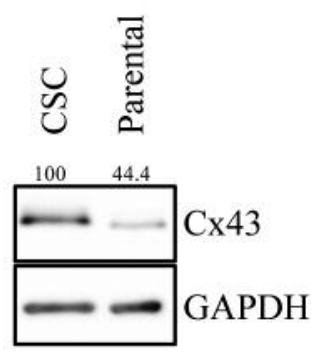

B

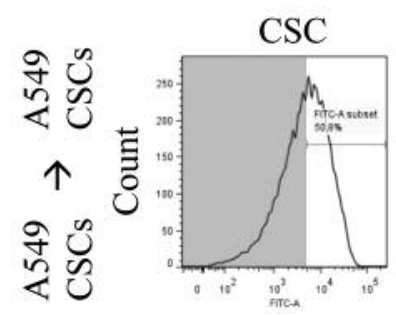

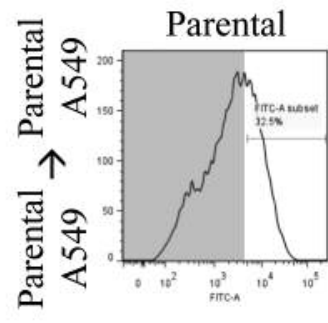

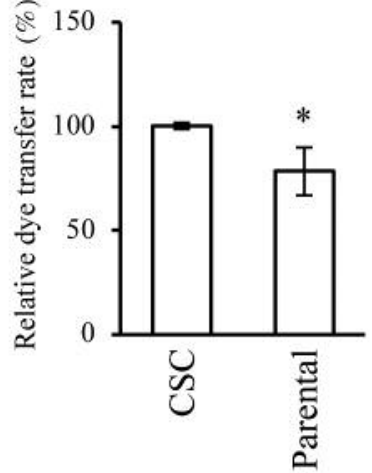

C

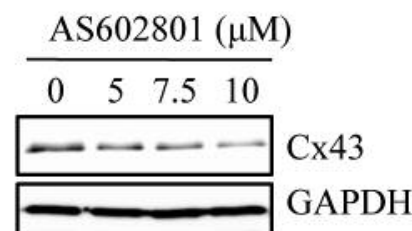

A549 CSC
D

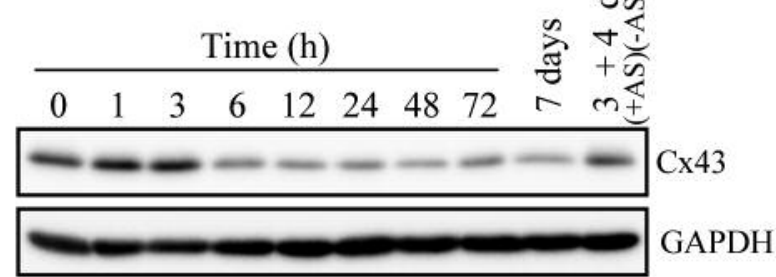

$\mathbf{E}$

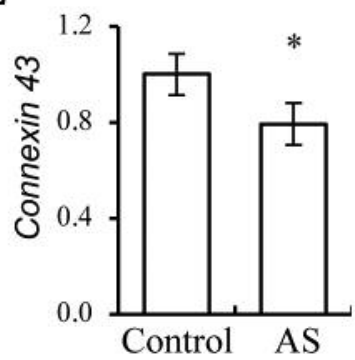

Figure 4. AS602801 suppressed connexin 43 (Cx43) expression in A549 CSCs. (A) A549 CSCs and parental A549 cell lysates were subjected to immunoblot analysis of CX43 and GAPDH. Immunoblot signals were quantified via densitometry and normalized to the values obtained in CSCs. (B) Histograms and quantification of dye transfer between A549 CSCs and A549 CSCs or between parental A549 and parental A549 cells. (C) A549 CSCs were treated with the indicated concentrations of AS602801 for three days and subjected to immunoblot analyses of the indicated proteins. (D) A549 CSCs were treated with $7.5 \mu M$ AS602801 for the indicated time periods and then subjected to immunoblot analyses of the indicated proteins. (E) A549 CSCs were treated without (Control) or with $7.5 \mu M$ AS602801 for $24 \mathrm{~h}$ and then subjected to RT-PCR. The graph shows the expression levels of CX43 mRNA, using ImageJ ( $n=3$ per group). Representative histograms are shown. Data are shown as means $\pm S . D$. from triplicate samples of a representative experiment repeated with similar results. ${ }^{*} p<0.05$.

In summary, AS602801 suppressed cell-cell communications via GJs and $\mathrm{Cx} 43$ expression level. To the best of our knowledge, this is the first such report. The Cx43 expression level and cell-cell communication via GJs were higher in CSCs than in the parental cells. Our findings suggest that AS602801 can serve as a novel agent against brain metastasis of lung cancer by inhibiting GJs in CSCs.

\section{Conflicts of Interest}

The aAuthors declare no conflict of interest in regard to this study.

\section{Acknowledgements}

The Authors thank Ms. Asuka Sugai for her secretarial contributions to this study. This work was supported by Grants-in-Aid for Scientific Research, for Challenging Exploratory Research from the Ministry of Education, Culture, Sports, Science and Technology of Japan (16K07160).

\section{References}

1 Gavrilovic IT and Posner JB: Brain metastases: epidemiology and pathophysiology. J Neurooncol 75: 5-14, 2005.

2 Lee GH, Jang B, Choi HS, Kim HJ, Park JH, Jeon YC, Carp RI, Kim YS and Choi EK: Upregulation of Connexin 43 Expression Via C-Jun N-Terminal Kinase Signaling in Prion Disease. J Alzheimers Dis 49: 1005-1019, 2016.

3 Sun YW, Xu J, Zhou J and Liu WJ: Targeted drugs for systemic therapy of lung cancer with brain metastases. Oncotarget 9: 5459-5472, 2018.

4 D’Antonio C, Passaro A, Gori B, Del Signore E, Migliorino MR, Ricciardi S, Fulvi A and de Marinis F: Bone and brain metastasis in lung cancer: recent advances in therapeutic strategies. Ther Adv Med Oncol 6: 101-114, 2014.

5 Chen Q, Boire A, Jin X, Valiente M, Er EE, Lopez-Soto A, Jacob L, Patwa R, Shah H, Xu K, Cross JR and Massague J: Carcinoma-astrocyte gap junctions promote brain metastasis by cGAMP transfer. Nature 533: 493-498, 2016.

6 Wasilewski D, Priego N, Fustero-Torre C and Valiente M: Reactive Astrocytes in Brain Metastasis. Front Oncol 7: 298, 2017. 
7 Aasen T, Mesnil M, Naus CC, Lampe PD and Laird DW: Gap junctions and cancer: communicating for 50 years. Nat Rev Cancer 16: 775-788, 2016.

8 Kim SJ, Kim JS, Park ES, Lee JS, Lin Q, Langley RR, Maya M, He J, Kim SW, Weihua Z, Balasubramanian K, Fan D, Mills GB, Hung MC and Fidler IJ: Astrocytes upregulate survival genes in tumor cells and induce protection from chemotherapy. Neoplasia 13: 286-298, 2011.

9 Lin Q, Balasubramanian K, Fan D, Kim SJ, Guo L, Wang H, Bar-Eli M, Aldape KD and Fidler IJ: Reactive astrocytes protect melanoma cells from chemotherapy by sequestering intracellular calcium through gap junction communication channels. Neoplasia 12: 748-754, 2010.

10 Chiou SH, Wang ML, Chou YT, Chen CJ, Hong CF, Hsieh WJ, Chang HT, Chen YS, Lin TW, Hsu HS and Wu CW: Coexpression of Oct4 and Nanog enhances malignancy in lung adenocarcinoma by inducing cancer stem cell-like properties and epithelial-mesenchymal transdifferentiation. Cancer Res 70: 10433-10444, 2010.

11 Singh S and Chellappan S: Lung cancer stem cells: Molecular features and therapeutic targets. Mol Aspects Med 39: 50-60, 2014.

12 Zibara K, Awada Z, Dib L, El-Saghir J, Al-Ghadban S, Ibrik A, El-Zein N and El-Sabban M: Anti-angiogenesis therapy and gap junction inhibition reduce MDA-MB-231 breast cancer cell invasion and metastasis in vitro and in vivo. Sci Rep 5: 12598, 2015.

13 Ferrandi C, Richard F, Tavano P, Hauben E, Barbie V, Gotteland JP, Greco B, Fortunato M, Mariani MF, Furlan R, Comi G, Martino $G$ and Zaratin PF: Characterization of immune cell subsets during the active phase of multiple sclerosis reveals disease and c-Jun N-terminal kinase pathway biomarkers. Mult Scler 17: 43-56, 2011.

14 Hussein M, Chai DC, Kyama CM, Mwenda JM, Palmer SS, Gotteland JP and D'Hooghe TM: c-Jun NH2-terminal kinase inhibitor bentamapimod reduces induced endometriosis in baboons: an assessor-blind placebo-controlled randomized study. Fertil Steril 105: 815-824.e815, 2016.

15 Palmer SS, Altan M, Denis D, Tos EG, Gotteland JP, Osteen KG, Bruner-Tran KL and Nataraja SG: Bentamapimod (JNK Inhibitor AS602801) Induces Regression of Endometriotic Lesions in Animal Models. Reprod Sci 23: 11-23, 2016.

16 Okada M, Kuramoto K, Takeda H, Watarai H, Sakaki H, Seino S, Seino M, Suzuki S and Kitanaka C: The novel JNK inhibitor AS602801 inhibits cancer stem cells in vitro and in vivo. Oncotarget 7: 27021-27032, 2016.

17 Okada M, Shibuya K, Sato A, Seino S, Watanabe E, Suzuki S, Seino $M$ and Kitanaka $C$ : Specific role of JNK in the maintenance of the tumor-initiating capacity of A549 human non-small cell lung cancer cells. Oncol Rep 30: 1957-1964, 2013 .

18 Kuramoto K, Suzuki S, Sakaki H, Takeda H, Sanomachi T, Seino S, Narita Y, Kayama T, Kitanaka C and Okada M: Licochalcone A specifically induces cell death in glioma stem cells via mitochondrial dysfunction. FEBS Open Bio 7: 835-844, 2017.
19 Okada M, Takeda H, Sakaki H, Kuramoto K, Suzuki S, Sanomachi T, Togashi K, Seino S and Kitanaka C: Repositioning CEP-1347, a chemical agent originally developed for the treatment of Parkinson's disease, as an anti-cancer stem cell drug. Oncotarget 8: 94872-94882, 2017.

20 Bennett MV, Contreras JE, Bukauskas FF and Saez JC: New roles for astrocytes: gap junction hemichannels have something to communicate. Trends Neurosci 26: 610-617, 2003.

21 Gaspar LE, Chansky K, Albain KS, Vallieres E, Rusch V, Crowley JJ, Livingston RB and Gandara DR: Time from treatment to subsequent diagnosis of brain metastases in stage III non-small-cell lung cancer: a retrospective review by the Southwest Oncology Group. J Clin Oncol 23: 2955-2961, 2005.

22 Gaspar LE, Scott C, Murray K and Curran W: Validation of the RTOG recursive partitioning analysis (RPA) classification for brain metastases. Int J Radiat Oncol Biol Phys 47: 1001-1006, 2000.

23 Giaume C, Koulakoff A, Roux L, Holcman D and Rouach N: Astroglial networks: a step further in neuroglial and gliovascular interactions. Nat Rev Neurosci 11: 87-99, 2010.

24 Bennett BL: c-Jun N-terminal kinase-dependent mechanisms in respiratory disease. Eur Respir J 28: 651-661, 2006.

25 Gangoso E, Thirant C, Chneiweiss H, Medina JM and Tabernero A: A cell-penetrating peptide based on the interaction between c-Src and connexin43 reverses glioma stem cell phenotype. Cell Death Dis 5: e1023, 2014.

26 Yu SC, Xiao HL, Jiang XF, Wang QL, Li Y, Yang XJ, Ping YF, Duan JJ, Jiang JY, Ye XZ, Xu SL, Xin YH, Yao XH, Chen JH, Chu WH, Sun W, Wang B, Wang JM, Zhang X and Bian XW: Connexin 43 reverses malignant phenotypes of glioma stem cells by modulating E-cadherin. Stem Cells 30: 108-120, 2012.

27 Jinn $\mathrm{Y}$ and Inase N: Connexin 43, E-cadherin, beta-catenin and ZO-1 expression, and aberrant methylation of the connexin 43 gene in NSCLC. Anticancer Res 30: 2271-2278, 2010.

28 Xu HT, Li QC, Zhang YX, Zhao Y, Liu Y, Yang ZQ and Wang EH: Connexin 43 recruits E-cadherin expression and inhibits the malignant behaviour of lung cancer cells. Folia Histochem Cytobiol 46: 315-321, 2008.

29 Gao FH, Wang Q, Wu YL, Li X, Zhao KW and Chen GQ: c-Jun $\mathrm{N}$-terminal kinase mediates AML1-ETO protein-induced connexin-43 expression. Biochem Biophys Res Commun 356: 505-511, 2007.

30 Munoz JL, Rodriguez-Cruz V, Greco SJ, Ramkissoon SH, Ligon KL and Rameshwar P: Temozolomide resistance in glioblastoma cells occurs partly through epidermal growth factor receptormediated induction of connexin 43. Cell Death Dis 5: e1145, 2014.

31 Shyu KG, Wang BW, Yang YH, Tsai SC, Lin S and Lee CC: Amphetamine activates connexin 43 gene expression in cultured neonatal rat cardiomyocytes through JNK and AP-1 pathway. Cardiovasc Res 63: 98-108, 2004.

Received July 16, 2018

Revised July 292018

Accepted July 30, 2018 